\title{
Morphological and molecular characterization of Termitomyces (Lyophyllaceae, Agaricales) in Thailand
}

\author{
NICHAREE JANNUAL ${ }^{1}$, MINGKWAN NIPITWATTANAPHON ${ }^{1}$, SASITORN HASIN ${ }^{2}$, \\ THARNRAT KAEWGRAJANG ${ }^{3, \varphi}$ \\ ${ }^{1}$ Department of Genetics, Faculty of Science, Kasetsart University. 50 Ngamwongwan Rd, Lat Yao, Chatuchak, Bangkok 10900, Thailand. \\ ${ }^{2}$ College of Innovative Management, Valaya Alongkorn Rajabhat University under the Royal Patronage. 100 years Somdej Prasrinakarin, Klong Luang, \\ Pathumthani 13180, Thailand. \\ ${ }^{3}$ Department of Forest Biology, Faculty of Forestry, Kasetsart University. 50 Ngamwongwan Rd, Lat Yao, Chatuchak, Bangkok 10900, Thailand. \\ Tel.: +66-257-90176, Fax.: +66-294-28107, `email: ffortrk@ku.ac.th
}

Manuscript received: 11 February 2020. Revision accepted: 13 May 2020.

\begin{abstract}
Jannual N, Nipitwattanaphon M, Hasin S, Kaewgrajang T. 2020. Morphological and molecular characterization of Termitomyces (Lyophyllaceae, Agaricales) in Thailand. Biodiversitas 21: 2481-2491. Termitomyces is considered to be a highly prized delicacy collected both for home consumption and for sale in local markets. Although the taxonomic information about this genus is well known in Africa, the identification of Termitomyces species in Thailand is unclear. Therefore, this study presented an assessment of phylogenetic relationships in the genus Termitomyces by means of sequencing of the ITS1-5.8S-ITS2 region and the species characterization by combination of morphological data and molecular data. Total of 61 Termitomyces specimens was collected from a variety of geographical localities of Thailand. They were classified into six species, including T. clypeatus, T. cylindricus, T. fuliginosus, T. heimii, T. microcarpus, and T. striatus based on morphological characteristics combining with molecular characteristics. This is the first diagnostic key of Thai Termitomyces.
\end{abstract}

Keywords: Diversity, morphology, termite mushroom, termitophilic Agaricales, taxonomy

Abbreviations: ACACIA: Acacia spp. plantation; DDF: dry dipterocarp forest; DEF: dry evergreen forest; EUCA: Eucalyptus plantation; HOPEA: Hopea odorata plantation; ITS: Internal transcribed spacer; MP: Maximum parsimony; TEAK: Teak plantation; TRF: tropical rain forest

\section{INTRODUCTION}

Thailand located in the tropical region, which has high diversity of flora and fauna, especially mushrooms (Tanticharoen 2004). Hyde et al. (2018) who reported that high species diversity of mushrooms are found in the northern part of Thailand and up to $93 \%$ of the mushrooms are novel species. Recently, Sangwanit et al. (2013) reported 2,575 species in the checklist of mushrooms in Thailand. Amongst these, species in the genus Termitomyces Heim are one of the popular wild edible mushrooms group in Thailand. The genus is one of highly prized delicacy collected both for home consumption and for sale in local markets or along roadside (Pegler and Vanhaecke 1994).

Termitomyces was firstly introduced by Roger Heim in 1942 to "termitophilic Agaricales" group associated with termite nests. Its various species are distributed throughout equatorial and Southern Africa (Otieno 1964; Mossebo et al. 2002), America (Tibuhwa et al. 2010), Europe (Olila et al. 2007), and Southeast Asia (Pegler and Vanhaecke 1994; Wei \& Yao 2003; Wei et al. 2003, 2004, 2006; Tang et al. 2006a, 2006b). Since 1942, about 30 Termitomyces species have so far been represented worldwide (Kirk et al. 2008). Currently, 92 different names for species, subspecies, and varieties are available in literature (www.indexfungorum.org).

Termitomyces species in Thailand was first reported by Bel P.J and Pataragetvit S., which did the field survey from Kanchanaburi province western Thailand upward along with the Kwai river to Chiangmai province northern Thailand during 1978-1979. From this survey, four species, T. clypeatus, T. fuliginosus, T. globulus, T. mammiforius were recorded (Bels and Pataragetvit 1982). In 1994, three species, T. aurantiacus, T. clypeatus, and T. globulus, were described from specimens collected at Chiang Mai and Kanchanaburi provinces, Thailand (Pegler and Vanhaecke 1994). After that, several field surveys for Termitomyces had done by Thai mycologists and now 18 species of Thai Termitomyces had been reported (The Royal Society 1996; Pitchayangkul 1998; Sangwanit et al. 2013). However, most of reports were identified the mushrooms based on morphological characteristics according to constructing identification Key from Africa. Although molecular technique has been accepted as useful technique for mushroom classification, only a few studies used morphological characteristics together with molecular techniques for identification. Taprab et al. (2002) extracted DNA from nodules and classified into eight groups of Termitomyces species based on internal transcribed sequences (ITS) but no evidence of mushroom 
morphological support. Additionally, Kosakul et al. (2007) used isozyme markers to determine the genetic diversity of T. auranticus, T. entolomoides, T. heimii, T. clypeatus and T. cylindricus from 20 Termitomyces species collected from the central region of Thailand but also without description of morphological characteristics. Only the report of Sawhasan et al. (2010) classified nine species of Termitomyces collected in Kanchanaburi province based on morphological characteristics and ITS1-5.8S-ITS2 rDNA sequences. It was shown that although the taxonomic information about this genus is well known in Africa, the identification of Termitomyces species in Thailand is unclear because there are some morphological variations from the description were found, especially in T. clypeatus, such as acutely spiniform and color of perforatorium, depended on environmental factors (Sawhasan et al. 2011). Thus, it could lead to misidentification and most of the samples remained unidentified. To overcome the difficulty of identifying species of this genus with morphology, molecular techniques should be combined with morphological characteristics to provide accurate species identification (Siddiquee et al. 2012). ITS (Internal transcribed spacer) region of the ribosomal DNA gene (rDNA) has been widely used for fungal classification and phylogenic studies (Hajibabaei et al. 2007; Seifert 2009; Bellemain et al. 2010; Hibbett et al. 2016; $\mathrm{Xu}$ and Adamowicz 2016; Raja et al. 2017). ITS combined with macroscopic and microscopic features to identify Termitomyces mushrooms has been done in many studies (Hofstetter et al. 2002; Rouland-Lefèvre et al. 2002; Sawhasan et al. 2011; Hussain et al. 2015; Raja et al. 2017). However, currently, very few presumptive sequence data of Termitomyces from Thailand are available making it difficult to use identify by DNA sequences. Thus, it is important to have the diagnostic key specific to Thai Termitomyces together with molecular study to allow any comparison between studies and to facilitate future identification by using DNA barcoding method. Consequently, the focus of this study was to identify Termitomyces species collected from various geographical localities of Thailand based on macroscopic features, microscopic features, and DNA barcoding, and to provide description of Termitomyces species with a diagnostic key.

\section{MATERIALS AND METHODS}

\section{Sampling sites and sample collections}

Termitomyces specimens were collected from a variety of geographical localities of Thailand (Table 1). The collection of this genus was carried out from 2015 to 2018 during August - October (the best time for collecting Termitomyces). The fruiting bodies were photographed, carefully examined by digging the soil to get complete pseudorhiza, and then removed the soil on the surface of pseudorhiza. The specimens were kept in a wax paper and brought to laboratory for observing macroscopic and microscopic features. Fruiting bodies for each isolate are maintained in the herbarium at the Forest Pathology Laboratory, Department of Forest Biology, Faculty of Forestry, Kasetsart University (Table 1).

\section{Macroscopic and microscopic identification}

Before oven-drying $\left(45^{\circ} \mathrm{C}\right)$, the macroscopic features were recorded in detail and were then made the spore print following Largent (1973). All the dried specimens were kept in silica gel for microscopic features and molecular works. Thin sections of dried specimens, including lamellae, pileal context, and partial veils were done by a free-hand section. The observation of microscopic features including size of basidiospores, basidia, hymenophoral trama, pileal context, and cutis was done under the compound microscope (Zeiss Axioskop 40, magnification 1000x) following Largent et al. (1977). At least 30 basidiospores, 20 basidia, and 20 cystidia of each specimen were measured. The specimens were identified based on diagnostic macroscopic and microscopic features examination (Pegler and Vanhaecke 1994; Wei et al. 2004; 2006; 2009; Mossebo et al. 2009; Sawhasan et al. 2011; Karun et al. 2013).

\section{Molecular identification}

Samples for DNA extraction were excised from dry or fresh basidiomes. Genomic DNA was isolated using FavorPrep $^{\mathrm{TM}}$ Tissue Genomic DNA Extraction Mini Kit (Favorgen Inc., Taiwan). The ITS regions were amplified with Termitomyces specific primer, ITS1FT (5'GTTTTCAACCACCTGTGCAC-3') (Nobre et al. 2010), ITS4 (5'-TCCTCCGCTTATTGATATGC-3') (White et al. 1990) and LR7 (5'-TACTACCACCAAGATCT-3') (Vilgalys and Hester 1990).

PCR reaction was performed in $50 \mu \mathrm{L}$ containing $\sim 20$ ng of DNA template, $1 \mathrm{X}$ PCR buffer, $0.5 \mathrm{mM}$ dNTP, 5 $\mathrm{mM} \mathrm{MgCl}_{2}, 0.625 \mu \mathrm{M}$ of each primer and $1.25 \mathrm{U}$ Taq DNA polymerase (Apsalagen, Thailand). PCR condition includes pre-denaturation at $95^{\circ} \mathrm{C}$ for 3 min followed by 40 cycles of denaturation at $95^{\circ} \mathrm{C}$ for $30 \mathrm{~s}$, annealing at $45^{\circ} \mathrm{C}$ for $30 \mathrm{~s}$, and extension at $72^{\circ} \mathrm{C}$ for $60-90 \mathrm{~s}$, and final extension at $72^{\circ} \mathrm{C}$ for $5 \mathrm{~min}$. PCR products were checked by $1 \%$ agarose gel electrophoresis and purified with FavorPrep $^{\mathrm{TM}}$ GEL/PCR Purification Kit (Favorgen Inc., Taiwan). After that, purified PCR products were sent to Macrogen Inc. (Korea) for Sanger sequencing. All sequences data in this study were deposited in Genbank (MN160255- MN160316) (Table 1).

Other Termitomyces sequences were retrieved from GenBank for phylogenetic analysis together with sequences from this study. All sequences were aligned using MAFFT (Nakamura et al. 2018) and manually edited the alignment using Bioedit program, version 7.2.5 (Hall 1999). Phylogenetic trees were reconstructed by MEGA7 program (Kumar et al. 2016) using Maximum parsimony (MP) method. Number of bootstraps was set to 1000 replicates. 
Table 1. List of Termitomyces specimens included in this study

\begin{tabular}{|c|c|c|c|c|c|}
\hline Code & $\begin{array}{c}\text { Herbarium } \\
\text { no. }\end{array}$ & $\begin{array}{l}\text { Vegetation/ } \\
\text { Forest type }\end{array}$ & Sampling site & $\begin{array}{c}\text { GenBank } \\
\text { accession no. }\end{array}$ & Length (bp) \\
\hline M02 css 1 & TERM001 & DEF & Wang NamKhiao, Nakhon Ratchasrima & MN160255 & 1942 \\
\hline M02 css 2 & TERM002 & DEF & Wang NamKhiao, Nakhon Ratchasrima & MN160256 & 1953 \\
\hline M03 & TERM003 & DDF & Wang NamKhiao, Nakhon Ratchasrima & MN160257 & 1938 \\
\hline M05 & TERM004 & DEF & Wang NamKhiao, Nakhon Ratchasrima & MN160258 & 721 \\
\hline M012 & TERM005 & HOPEA & Wang NamKhiao, Nakhon Ratchasrima & MN160259 & 1229 \\
\hline M013 & TERM006 & HOPEA & Wang NamKhiao, Nakhon Ratchasrima & MN160260 & 1967 \\
\hline M015 & TERM007 & DEF & Wang NamKhiao, Nakhon Ratchasrima & MN160261 & 186 \\
\hline M016 & TERM008 & HOPEA & Wang NamKhiao, Nakhon Ratchasrima & MN160262 & 669 \\
\hline M017 & TERM009 & DDF & Wang NamKhiao, Nakhon Ratchasrima & MN160263 & 617 \\
\hline M018 & TERM010 & DDF & Wang NamKhiao, Nakhon Ratchasrima & MN160264 & 624 \\
\hline M019 & TERM011 & DDF & Wang NamKhiao, Nakhon Ratchasrima & MN160265 & 668 \\
\hline M023 & TERM012 & EUCA & Wang NamKhiao, Nakhon Ratchasrima & MN160266 & 624 \\
\hline M024 & TERM013 & plantation & Wang NamKhiao, Nakhon Ratchasrima & MN160267 & 1966 \\
\hline M026 & TERM014 & EUCA & Wang NamKhiao, Nakhon Ratchasrima & MN160268 & 171 \\
\hline EU611 & TERM015 & EUCA & Wang NamKhiao, Nakhon Ratchasrima & MN160269 & 1897 \\
\hline EU612 & TERM016 & EUCA & Wang NamKhiao, Nakhon Ratchasrima & MN160270 & 1897 \\
\hline EU613 & TERM017 & EUCA & Wang NamKhiao, Nakhon Ratchasrima & MN160271 & 1897 \\
\hline Mix611 & TERM018 & plantation & Wang NamKhiao, Nakhon Ratchasrima & MN160272 & 1856 \\
\hline Mix612 & TERM019 & plantation & Wang NamKhiao, Nakhon Ratchasrima & MN160273 & 1212 \\
\hline Mix613 & TERM020 & plantation & Wang NamKhiao, Nakhon Ratchasrima & MN160274 & 1898 \\
\hline Mix614 & TERM021 & plantation & Wang NamKhiao, Nakhon Ratchasrima & MN160275 & 1214 \\
\hline Mix615 & TERM022 & plantation & Wang NamKhiao, Nakhon Ratchasrima & MN160276 & 1898 \\
\hline SKR00 & TERM023 & DEF & Wang NamKhiao, Nakhon Ratchasrima & MN160277 & 159 \\
\hline SKR01 DEF & TERM024 & DEF & Wang NamKhiao, Nakhon Ratchasrima & MN160278 & 543 \\
\hline SRK02 DEF2 & TERM025 & DEF & Wang NamKhiao, Nakhon Ratchasrima & MN160279 & 1930 \\
\hline SKR03 Acacia & TERM026 & ACACIA & Wang NamKhiao, Nakhon Ratchasrima & MN160280 & 1859 \\
\hline SKR04 DEF & TERM027 & DEF & Wang NamKhiao, Nakhon Ratchasrima & MN160281 & 186 \\
\hline SKR05 Acacia & TERM028 & ACACIA & Wang NamKhiao, Nakhon Ratchasrima & MN160282 & 984 \\
\hline SKR07 DEF2 & TERM029 & DEF & Wang NamKhiao, Nakhon Ratchasrima & MN160283 & 1894 \\
\hline SKR08 DEF2 & TERM030 & DEF & Wang NamKhiao, Nakhon Ratchasrima & MN160284 & 1904 \\
\hline SKR09 Acacia & TERM031 & ACACIA & Wang NamKhiao, Nakhon Ratchasrima & MN160285 & 186 \\
\hline SKR071 & TERM032 & DEF & Wang NamKhiao, Nakhon Ratchasrima & MN160286 & 183 \\
\hline SKR086 DEF9 & TERM033 & DEF & Wang NamKhiao, Nakhon Ratchasrima & MN160287 & 1089 \\
\hline SKR612 & TERM034 & DEF & Wang NamKhiao, Nakhon Ratchasrima & MN160288 & 1858 \\
\hline SKR613 & TERM035 & DEF & Wang NamKhiao, Nakhon Ratchasrima & MN160289 & 1832 \\
\hline SKR614 & TERM036 & DEF & Wang NamKhiao, Nakhon Ratchasrima & MN160290 & 1879 \\
\hline TA1 & TERM037 & DDF & Wang NamKhiao, Nakhon Ratchasrima & MN160291 & 301 \\
\hline TA2 & TERM038 & DDF & Wang NamKhiao, Nakhon Ratchasrima & MN160292 & 280 \\
\hline TA3 & TERM039 & DDF & Wang NamKhiao, Nakhon Ratchasrima & MN160293 & 705 \\
\hline TAP & TERM040 & DDF & Wang NamKhiao, Nakhon Ratchasrima & MN160294 & 424 \\
\hline TAT & TERM041 & DDF & Wang NamKhiao, Nakhon Ratchasrima & MN160295 & 421 \\
\hline Ter_Mix & TERM042 & plantation & Wang NamKhiao, Nakhon Ratchasrima & MN160296 & 1881 \\
\hline Ter1M & TERM043 & DEF & Wang NamKhiao, Nakhon Ratchasrima & MN160297 & 1938 \\
\hline Ter2M & TERM044 & DEF & Wang NamKhiao, Nakhon Ratchasrima & MN160298 & 691 \\
\hline Ter3M & TERM045 & DEF & Wang NamKhiao, Nakhon Ratchasrima & MN160299 & 967 \\
\hline Ter4M & TERM046 & DEF & Wang NamKhiao, Nakhon Ratchasrima & MN160300 & 1952 \\
\hline Ter5 & TERM047 & DEF & Wang NamKhiao, Nakhon Ratchasrima & MN160301 & 775 \\
\hline WNK611 & TERM048 & DEF & Wang NamKhiao, Nakhon Ratchasrima & MN160302 & 1900 \\
\hline WNK612 & TERM049 & DEF & Wang NamKhiao, Nakhon Ratchasrima & MN160303 & 1900 \\
\hline WNK613 & TERM050 & DEF & Wang NamKhiao, Nakhon Ratchasrima & MN160304 & 1901 \\
\hline WNK614 & TERM051 & DEF & Wang NamKhiao, Nakhon Ratchasrima & MN160305 & 1577 \\
\hline WNK615 & TERM052 & DEF & Wang NamKhiao, Nakhon Ratchasrima & MN160306 & 1645 \\
\hline WNK617 & TERM053 & DEF & Wang NamKhiao, Nakhon Ratchasrima & MN160307 & 1656 \\
\hline WNK618 & TERM054 & DEF & Wang NamKhiao, Nakhon Ratchasrima & MN160308 & 1656 \\
\hline M08 & TERM055 & TEAK & Thong Pha Phum, Kanchanaburi & MN160309 & 1213 \\
\hline Ter1_Kan & TERM056 & TEAK & Thong Pha Phum, Kanchanaburi & MN160310 & 581 \\
\hline Ter6 & TERM057 & TRF & Kantang, Tang & MN160311 & 1222 \\
\hline Ter7 & TERM058 & TRF & Kantang, Tang & MN160312 & 1898 \\
\hline Ter8 & TERM059 & TRF & Kantang, Tang & MN160313 & 2001 \\
\hline Ter9 & TERM060 & TRF & Kantang, Tang & MN160314 & 2015 \\
\hline Ter10 & TERM061 & TRF & Kantang, Tang & MN160315 & 2003 \\
\hline M021 & TERM062 & DDF & Mueang, Sukhothai & MN160316 & 618 \\
\hline
\end{tabular}

Note: DDF: dry dipterocarp forest; DEF: dry evergreen forest; TRF: tropical rain forest; EUCA: Eucalyptus plantation; TEAK: Teak plantation; ACACIA: Acacia spp. plantation; HOPEA: Hopea odorata plantation 


\section{RESULTS AND DISCUSSION}

\section{Molecular analysis}

MP tree showed the Termitomyces samples in this study were clustered together with sequences from GenBank (Figure 1). Six clades were clustered according to species. Nine Termitomyces samples identified as T. fuliginosus were grouped with $T$. fuliginosus sequences from other countries such as Viet Nam and China, T. eurrhizus, and Termitomyces sp. Group8 from Taprab et al. (2002). Fifteen Termitomyces samples identified as T. cylindricus were clustered with Termitomyces sp. Group2 and $T$. cylindricus from Thailand and Indonesia in the database. Similarly, 12 Termitomyces samples identified as $T$. microcarpus were grouped with Termitomyces sp. Group3 and T. microcarpus from Thailand and India. Likewise, TERM055 and TERM062 were also grouped with their identified species, T. heimii and T. clypeatus, respectively. On the other hand, Termitomyces samples that morphological characteristics confidently identified as $T$. striatus, showed no match with the only one ITS sequence of $T$. striatus in the database, and grouped with Termitomyces sp. Group7 from Taprab et al. (2002). Since there is only one ITS sequence but several $28 \mathrm{~S}$ sequences of $T$. striatus, we performed another phylogenetic tree of 28S from some Termitomyces species (Figure 2). The result from this tree showed that the samples identified as $T$. striatus in this study were related to other $28 \mathrm{~S}$ of $T$. striatus from GenBank but with some degree of intraspecific distance.

\section{Morphological description}

Termitomyces cylindricus S.C. He 1985 (Figure 3.A-F)

Pileus 5-7 cm diam., conic to convex then convexoapplanate to plane when mature, with an obtusely rounded perforatorium; surface white to cream, and yellow-brown at the center and paler toward margin, smooth and glabrous, margin straight, often radially splitting when mature. Lamellae free, white to cream and becoming pinkish when mature, $0.4 \mathrm{~cm}$ wide, crowded with lamellulae. Stipe 5-8.7 $\mathrm{cm}$ long, $0.7-1 \mathrm{~cm}$ wide, central, cylindrical to subcylindrical, surface white, smooth or longitudinally striate, solid fibrous. Annulus absent. Pseudorhiza 3-7 cm long, $0.3-0.5 \mathrm{~cm}$ thick, cylindrical to subcylindrical, surface white, smooth or longitudinally striate, solid. Spore deposit white. Basidiospores 5.5-10 x 4-7 $\mu \mathrm{m}$, ovoid to ellipsoid; smooth and thin-walled, inamyloid. Basidia 7.5-22.5 x 5-8 $\mu \mathrm{m}$, clavate, bearing four sterigmata, inamyloid. Hymenophoral trama regular, 55-70 $\mu \mathrm{m}$ wide, inamyloid. Cheilocystidia crowded, $20-46.3 \times 13-30 \mu \mathrm{m}$, clavate to pyriform, smooth and thin-walled, inamyloid. Pleurocystidia 20-47.5 x 10-28 $\mu \mathrm{m}$, similar to cheilocystidia Pileipellis a repent epicutis of narrow, radial hyphae, 1.5-2.5 $\mu \mathrm{m}$ diam.

Specimens examined:- Nakhon Ratchasrima: Wang Nam Khiao, Sakaerat environmental research station, in dry evergreen forest (TERM024, TERM025, TERM027,
TERM029, TERM030, TERM032, TERM033, TERM044, TERM045, TERM046), Nakhon Ratchasrima: Wang Nam Khiao, Sakaerat environmental research station, in Acacia sp. plantation (TERM026, TERM028, TERM031), Saraburi: Sao Hai, under Dipterocarpus alatus trees (TERM004), Nakhon Ratchasrima: Wang Nam Khiao, Sakaerat silviculture research station, dry dipterocarp forest (TERM039).

Termitomyces clypeatus R. Heim 1951 (Figure 3.G-J)

Pileus 6-10 cm diam., at first pointed conical then broadly convex to plane, with a dark prominent spiniform perforatorium; surface greyish brown to ochraceous brown, dark at the center and paler towards the margin when mature, fibrillose and silky, margin at first incurved then straight, often splitting. Lamellae free, white to cream, 0.8$1.0 \mathrm{~cm}$ wide, crowded with lamellulae. Stipe $6.5-12 \mathrm{~cm}$ long, 1-2 cm wide, central, cylindrical to subcylindrical, occasionally forming slightly thickening at stipe base, surface white with longitudinal fibrillose, solid. Annulus absent. Pseudorhiza 3-10 cm long, 0.3-1.0 cm thick, tapering towards the base, surface white to cream, smooth, solid. Spore deposit not found. Basidiospores 5-8 x 3-6 $\mu \mathrm{m}$, ovoid to ellipsoid; smooth and thin-walled, inamyloid. Basidia $12-20 \times 5-8 \mu \mathrm{m}$, clavate to subcylindric, bearing four sterigmata, inamyloid. Hymenophoral trama regular, up to $100 \mu \mathrm{m}$ wide, inamyloid. Cheilocystidia crowded, 17$18 \times 9-10 \mu \mathrm{m}$, clavate to pyriform, smooth and thin-walled, inamyloid. Pleurocystidia crowded, 23-42 x 9-30 $\mu \mathrm{m}$, similar to cheilocystidia Pileipellis a repent epicutis of narrow, radial hyphae, 2.0-4.0 $\mu \mathrm{m}$ diam.

Specimen examined:- Sukhothai: Mueang, Ban Kluai (TERM062).

\section{Termitomyces fuliginosus R. Heim 1942 Figure 3.K-P)}

Pileus 5-8 cm diam., conic to convex then convexoapplanate to plane when mature, with a spiniform at first and bluntly pointed perforatorium when mature; surface golden brown to brownish orange, radially fibrillose and shiny, margin at first incurved then straight, often splitting. Lamellae free, white to cream, $0.5 \mathrm{~cm}$ wide, crowded with lamellulae. Stipe $8-10 \mathrm{~cm}$ long, $1.0-1.3 \mathrm{~cm}$ wide, central, cylindrical or slightly bulbous at stipe base, surface cream to pale brown covering with pale brown fibrillose, solid fibrous. Annulus membranous ephemeral and often absent after the initial stage, attached to the upper of stipe. Pseudorhiza $10-20 \mathrm{~cm}$ long, $0.3-0.5 \mathrm{~cm}$ thick, cylindrical to slender, surface white, smooth or longitudinally striate, solid. Spore deposit white. Basidiospores 4.5-7 x 3-4.5 $\mu \mathrm{m}$, ellipsoid; smooth and thin-walled, inamyloid. Basidia 16$20 \times 6-8 \mu \mathrm{m}$, clavate, bearing four sterigmata, inamyloid. Hymenophoral trama regular, 50-72 $\mu \mathrm{m}$ wide, inamyloid. Cheilocystidia crowded, 18-24 x 10-11 $\mu \mathrm{m}$, clavate, smooth and thin-walled, inamyloid. Pleurocystidia scattered to rare, 20-30 $\times$ 8.5-11 $\mu \mathrm{m}$, similar to cheilocystidia. Pileipellis a repent epicutis of narrow, radial hyphae, 1.0-2.5 $\mu \mathrm{m}$ diam. 


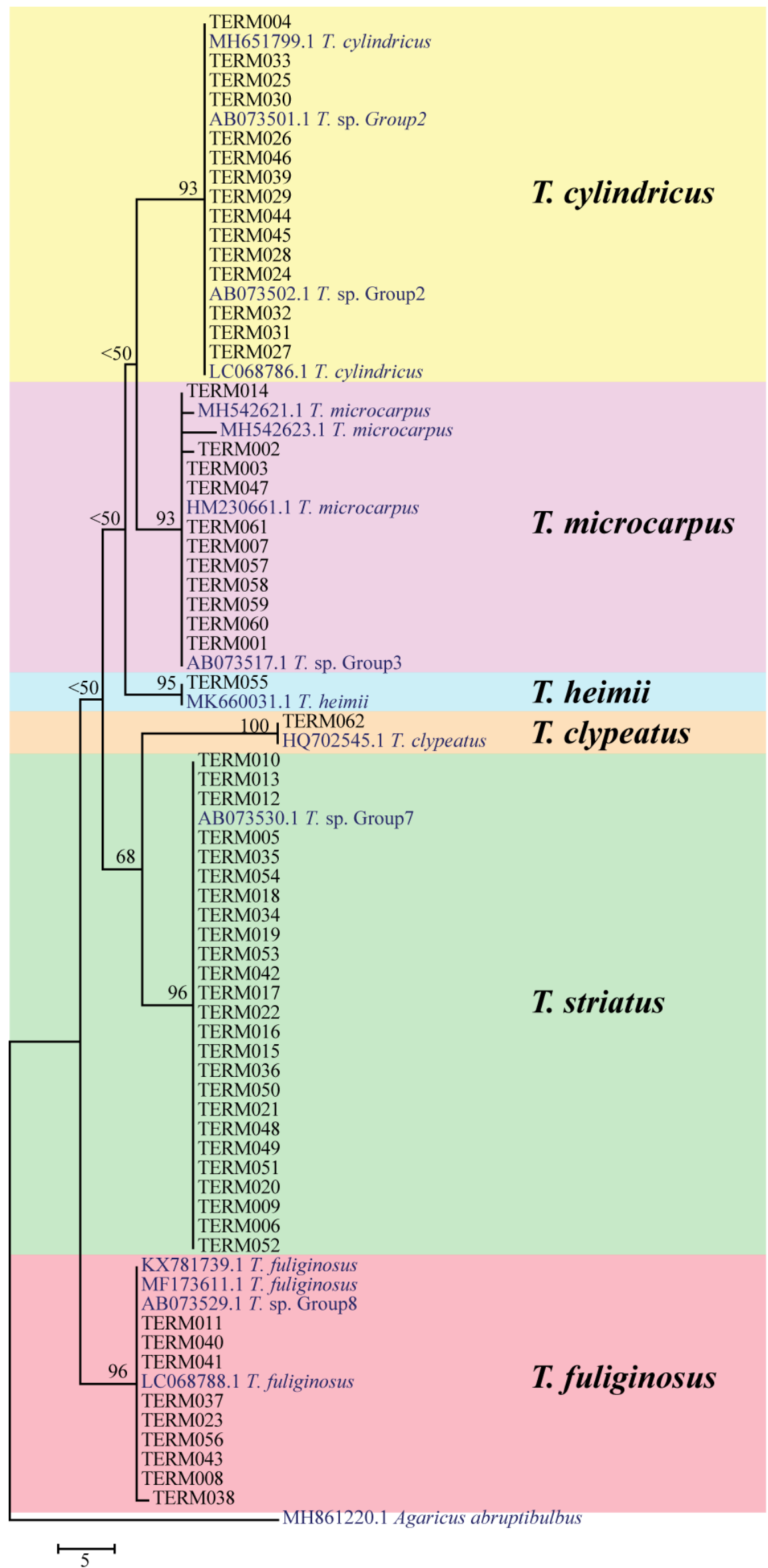

Figure 1. Phylogenetic tree based on ITS1-5.8S-ITS2 of Termitomyces sequences in this study and from GenBank (blue letter) using the Maximum parsimony method. Agaricus abruptibulbus is used as an outgroup. Numbers at the node are bootstrap scores of 1000 replicates. 


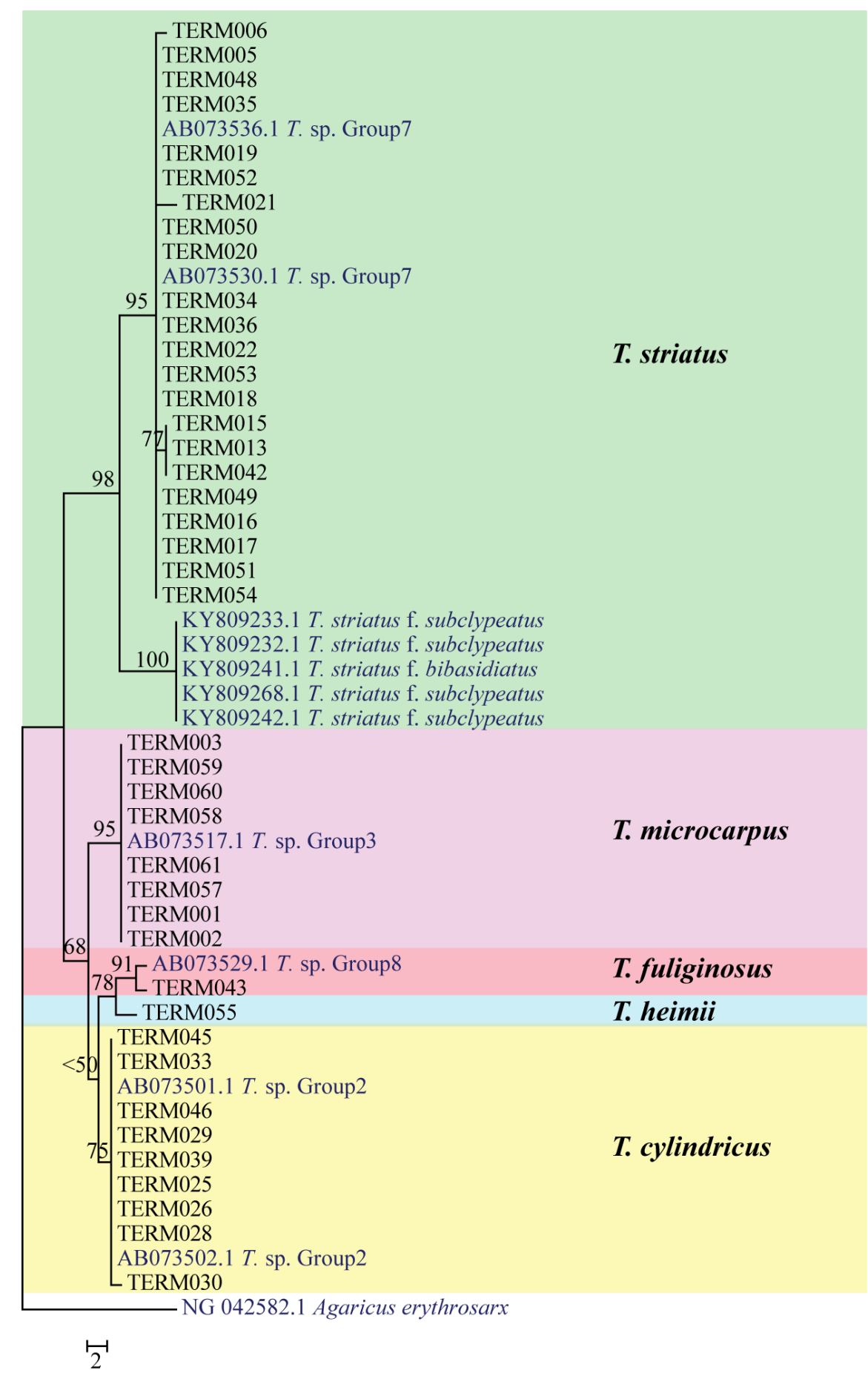

Figure 2. Phylogenetic tree based on 28S rDNA of Termitomyces sequences in this study and from GenBank (shown in blue letter) using the Maximum parsimony method. Agaricus erythrosarx is used as an outgroup. Numbers at the node are bootstrap scores of 1000 replicates

Specimens examined:- Nakhon Ratchasrima: Wang Nam Khiao, Sakaerat environmental research station, in dry evergreen forest (TERM043), Nakhon Ratchasrima: Wang Nam Khiao, Sakaerat silviculture research station, dry dipterocarp forest (TERM037, TERM038), Nakhon Ratchasrima: Wang Nam Khiao, Thaplan National park, in Hopea odorata plantation (TERM008), Nakhon Ratchasrima: Wang Nam Khiao, Wang Nam Khiao Forestry Student Training Station, in dry evergreen forest (TERM011), Nakhon Ratchasrima: Wang Nam Khiao,
Sakaerat environmental research station, in dry evergreen forest (TERM023), Nakhon Ratchasrima: Wang Nam Khiao, Sakaerat silviculture research station, dry dipterocarp forest (TERM040, TERM041), Kanchanaburi: Thong Pha Phum, teak plantation (TERM056).

\section{Termitomyces heimii Natarajan 1979 (Figure 4.A-C)}

Pileus $0.8-3.5 \mathrm{~cm}$ diam., parabolic to campanulate then plane to uplifted when mature, with rounded perforatorium; surface white to cream and dark at the center when mature, 
velar squamulose on surface, margin at first incurved then straight, often splitting. Lamellae free, white to creamy, 1 $\mathrm{mm}$ wide, crowded with lamellulae. Stipe $1.3-4 \mathrm{~cm}$ long, 0.5-2 cm wide, central, cylindrical to subcylindrical, surface white to cream, smooth and glabrous, solid. Annulus membranous at first, forming the persistent double annulus on the upper of stipe, surface white to cream. Pseudorhiza 7-20 cm long, 0.5-1.5 cm thick, cylindrical, surface white to cream, smooth, leathery, hollow. Spore deposit not found. Basidiospores 6-9.5 x 4-6 $\mu \mathrm{m}$, ovoid to ellipsoid; smooth and thin-walled, inamyloid. Basidia 1819 x 5-8 $\mu \mathrm{m}$, clavate to subcylindric, bearing four sterigmata, inamyloid. Hymenophoral trama regular, up to $100 \mu \mathrm{m}$ wide, inamyloid. Cheilocystidia crowded, 21-35 x 14-20 $\mu \mathrm{m}$, clavate to pyriform, smooth and thin-walled, inamyloid. Pleurocystidia rare, 20-34 x 11-18 $\mu \mathrm{m}$, similar to cheilocystidia Pileipellis a repent epicutis of narrow, radial hyphae, 2.0-4.5 $\mu \mathrm{m}$ diam.

Specimen examined:- Kanchanaburi: Thong Pha Phum, teak plantation (TERM055).

\section{Termitomyces microcarpus (Berk. \& Broome) R. Heim 1942 (Figure 4.D-F)}

Pileus 1.0-1.5 cm diam., conic then plane to uplifted when mature, with small obtuse perforatorium; surface white to cream and dark or yellowish-brown at the center when mature, smooth and glabrous, margin straight, often radially splitting when mature. Lamellae free, white to creamy, $1 \mathrm{~mm}$ wide, crowded with lamellulae. Stipe 4-6 cm long, 0.2-0.5 cm wide, central, cylindrical, surface white to cream, smooth and glabrous. Annulus absent. Pseudorhiza forming a root-like at stipe base under ground level. Spore deposit white. Basidiospores 4.5-8 x 3-5 $\mu \mathrm{m}$, ovoid to ellipsoid; smooth and thin-walled, inamyloid. Basidia 15$30 \times 5-10 \mu \mathrm{m}$, clavate to subcylindric, bearing four sterigmata, inamyloid. Hymenophoral trama regular, 50-60 $\mu \mathrm{m}$ wide, inamyloid. Cheilocystidia rare, 30-40 x 10-17.5 $\mu \mathrm{m}$, clavate to pyriform, smooth and thin-walled, inamyloid. Pleurocystidia rare, 25-35 x 10-17.5 $\mu \mathrm{m}$, similar to cheilocystidia. Pileipellis a repent epicutis of narrow, radial hyphae, 2.0-4.0 $\mu \mathrm{m}$ diam.

Specimens examined:- Nakhon Ratchasrima: Wang Nam Khiao, Sakaerat environmental research station, in dry evergreen forest (TERM001, TERM002), Nakhon Ratchasrima: Wang Nam Khiao, Wang Nam Khiao Forestry Student Training Station, in dry dipterocarp forest
(TERM003, TERM007, TERM014), Trang: Kachong, oil palm plantation (TERM047, TERM057, TERM058, TERM059, TERM060, TERM061).

\section{Termitomyces striatus (Beeli) R. Heim (Figure 4.G-K)}

Pileus 2-8 cm diam., conic to convex then plane when mature, with small and pointed perforatorium; surface light brown to greyish brown and dark at the center when mature, usually radially fibrillose, shiny, margin at first incurved then straight, often splitting. Lamellae free, white to creamy, 3-5 mm wide, crowded with lamellulae. Stipe $4.5-11 \mathrm{~cm}$ long, $0.5-1.5 \mathrm{~cm}$ wide, central, cylindrical to subcylindrical, occasionally swelling on its upper part and tapering at the base to form pseudorhiza, surface whitish, longitudinally striate, solid. Annulus absent. Pseudorhiza 7$20 \mathrm{~cm}$ long, $0.5-1.5 \mathrm{~cm}$ thick, tapering at the base; surface white to pale grey, smooth. Spore deposit pinkish. Basidiospores 5-8.5 x 4-6 $\mu \mathrm{m}$, ovoid to ellipsoid; smooth and thin-walled, inamyloid. Basidia 15-22 x 4-6 $\mu \mathrm{m}$, clavate to subcylindric, bearing four sterigmata, inamyloid. Hymenophoral trama regular, 40-100 $\mu \mathrm{m}$ wide, inamyloid. Cheilocystidia crowded, 20-32 x 6-17 $\mu \mathrm{m}$, clavate to pyriform, smooth and thin-walled, inamyloid. Pleurocystidia crowded, 17-32 x 6-16 $\mu \mathrm{m}$, similar to cheilocystidia, sometime subcylindrical or digitate. Pileipellis a repent epicutis of narrow, radial hyphae, 2.0$5.0 \mu \mathrm{m}$ diam.

Specimens examined:- Nakhon Ratchasrima: Wang Nam Khiao, ThaplanNational park, in Hopea odorata plantation (TERM005, TERM006), Nakhon Ratchasrima: Wang Nam Khiao, Wang Nam Khiao Forestry Student Training Station, in dry evergreen forest (TERM009, TERM010, TERM048, TERM051, TERM052, TERM054), Nakhon Ratchasrima: Wang Nam Khiao, Wang Nam Khiao Forestry Student Training Station, in Bamboo plantation (TERM049, TERM050, TERM053), Nakhon Ratchasrima: Wang Nam Khiao, Wang Nam Khiao Forestry Student Training Station, in mix forest tree species plantation (TERM013, TERM018, TERM019, TERM020, TERM021, TERM022, TERM042), Nakhon Ratchasrima: Wang Nam Khiao, Wang Nam Khiao Forestry Student Training Station, in Eucalyptus plantation (TERM012, TERM015, TERM016, TERM017), Nakhon Ratchasrima: Wang Nam Khiao, Sakaerat environmental research station, in dry evergreen forest (TERM034, TERM035, TERM036).

\section{Key to Termitomyces}

1a Mature pileus generally smaller than $2.0 \mathrm{~cm}$ diameter. T. microcarpus

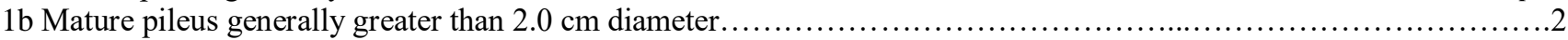

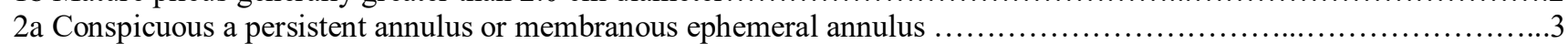

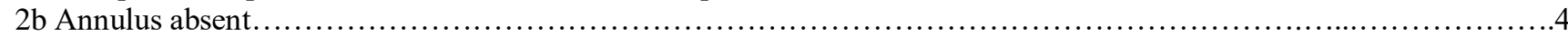
3a Pileus white to pale brown with rounded perforatorium, pseudorhiza leathery and hollow ....................T. heimii $3 \mathrm{~b}$ Pileus golden brown to brownish orange with bluntly pointed perforatorium, pseudorhiza freshy fibrous and solid

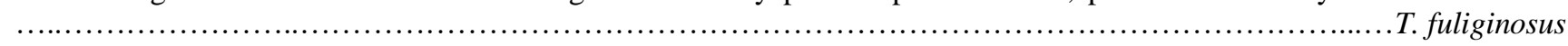
4a Pileus white to cream with obtusely rounded perforatorium, not spiniform..............................T. cylindricus

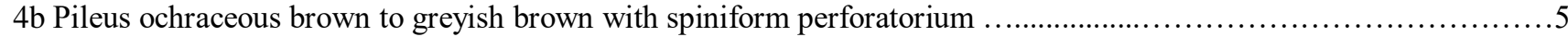
5a Perforatorium dark color, small pointed perforatorium, covering with radially striate ........................T. striatus 5 b Perforatorium dark color, prominent acutely perforatorium, smooth 

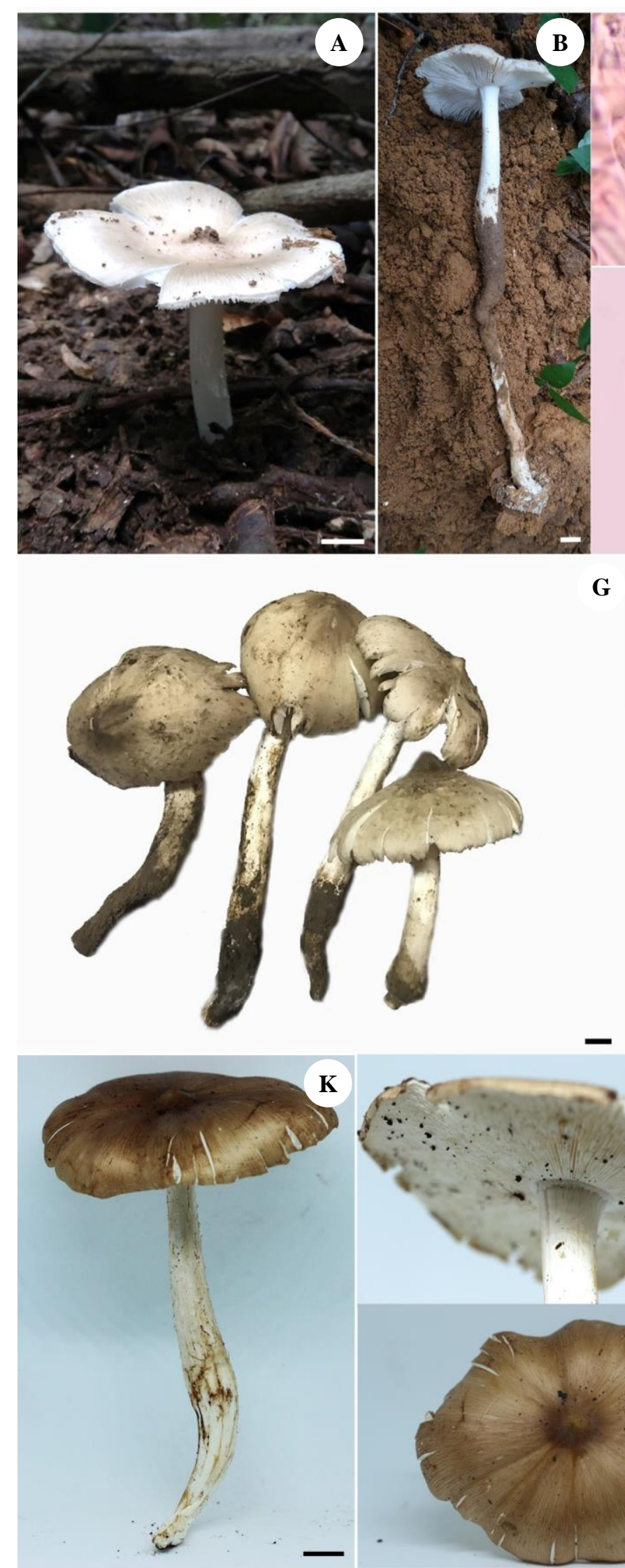

G

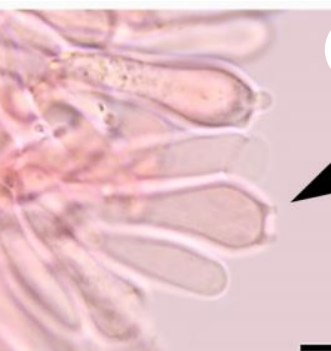

C
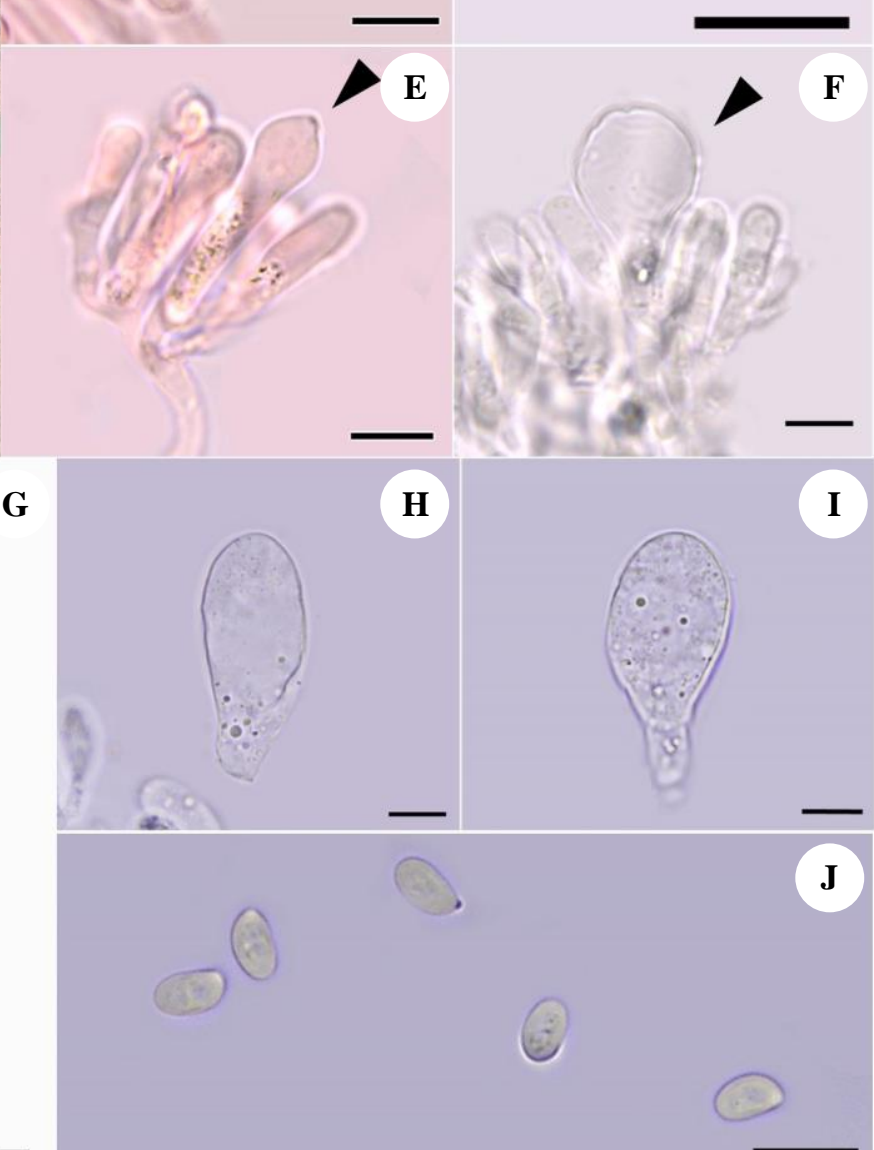

H

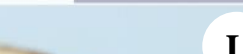

L

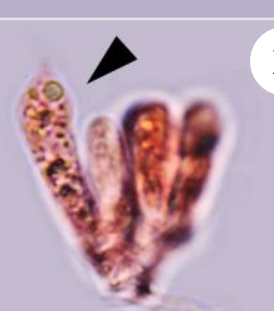

N
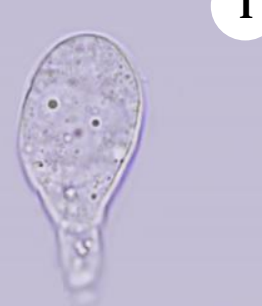

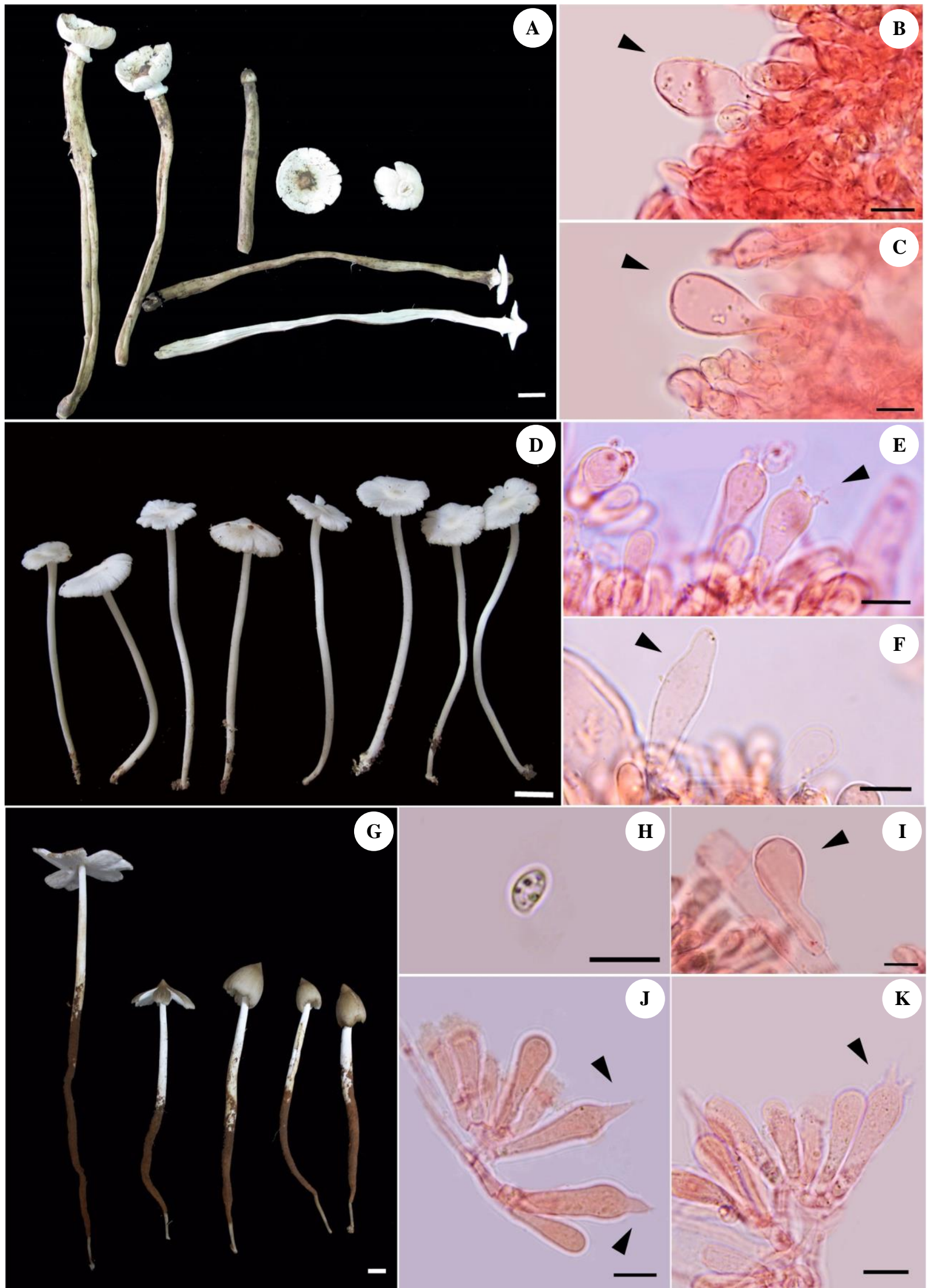


\section{Discussion}

Since 1978, the surveys of Thai Termitomyces have been done extensively. To date, 18 species of Termitomyces were reported, but most of which used only morphology including macroscopic features such as size and color of the pileus, and the presence of pseudorhiza (Bels and Pataragetvit 1982; The Royal Society 1996; Pitchayangkul 1998; Sangwanit et al. 2013). Nowadays, molecular techniques have been proved to be a handy tool in fungal classification because it provides more accurate species delimitation (Taylor et al. 2000; Siddiquee et al. 2012) and thus is required for analysis of very similar species (Tibuhwa et al. 2010). Since the absence of the description key and the differences of some morphologies in Thailand from previous studies, we provided here the first diagnostic key of six Thai Termitomyces species ( $T$. clypeatus, T. cylindricus, T. fuliginosus, T. heimii, $T$. microcarpus and T. striatus) from 62 samples. We used both morphological characteristics and molecular characteristics, because using only molecular techniques with unidentified species or without the accuracy of species identification by morphological identification can be problematic. Additionally, we investigated the evolutionary relationship of them based on phylogenetic analyses of ITS1-5.8s-ITS2 sequences (Figure 1).

Based on the description of our specimens, we found that the morphological characteristics of four Termitomyces species, T. fuliginosus, T. heimii, T. microcarpus and T. striatus, were corresponded with the previous description of Pegler and Vanhaecke (1994); Wei et al., (2009); Karun et al., (2013). Moreover, three of these four species ( $T$. fuliginosus, T. heimii, T. microcarpus) identified by DNA barcoding showed high \% identity (98-100\%) from BLAST results, matching with the same described species in the database. The absence of species matched with T. striatus may due to the deficiency of ITS sequences of Asian $T$. striatus in the database, because only one sequence from Congo was found and this sequence is rather distantly related to our T. striatus samples, suggesting that our species may be classified into different subspecies. The result from the $28 \mathrm{~S}$ tree (Figure 2) also supported that our T. striatus samples may belong to different subspecies with T. striatus from Congo since the samples were in the monophyletic clade with some genetic distance.

In addition, all samples were best hit with Termitomyces sequences collected from Thailand, which was studied by Taprab et al. (2002) despite no morphological characteristics of the submitted sequences to support. We also found that morphological characteristics of the other Termitomyces species in this study differed from previous studies, especially $T$. clyeatus. Our specimens of $T$. clypeatus do not have the acutely spiniform and dark color of perforatorium as mentioned by several reports (Pegler and Vanhaecke 1994; Wei et al. 2009; Karun et al. 2013). Likewise, the color of $T$. cylindricus in this study also differed from previous descriptions (Pegler and Vanhaecke 1994; Wei et al. 2009; Karun et al. 2013). Tibuhwa et al. (2010) reported that color is much used in identification keys to narrow the broad range of comparisons for different groups of species in the genus. Many variations of color are found within this genus from white-cream to grey, orange to brown, and rarely bluish to black between species. Thus, using pileus color might lead to misidentification of this genus. Phylogenetic tree of this study showed that most of our sequences were clustered according to species consistent with morphological identification. However, the sequence information of this genus from Thailand in the database was still little. Thus, here, we provided diagnostic keys for morphological identification together with the sequences of this study in the database for assisting more accurate identification and facilitating molecular identification by DNA barcoding of this genus in the future.

In conclusion, sixty-two samples of Termitomyces, which were collected from four provinces in Thailand, were identified based on morphological and molecular identification. These samples were classified into six species including T. microcarpus, T. heimii, T. fuliginosus, T. aurantiacus, T. striatus and T. clypeatus. Due to many variations in morphological features of this genus were found, the molecular identification is recommended to use together with morphological identification as the key provided here for more accurate identification of this genus.

\section{ACKNOWLEDGEMENTS}

This research was supported by the National Research Council of Thailand and the Biodiversity-Based Economy Development Office (Public Organization) (BEDO-NRCT No. 30/2015 and BEDO-NRCT No. 3/2017). We are also grateful to the Department of National park, Wildlife and Plant Conservation for supplying the necessary permit to collect specimens at Tablan national park, Nakhon Ratchasrima province (Doc. No. 0907.4/2533) on which the present study is based and without which it could not have been completed.

\section{REFERENCES}

Bellemain E, Carlsen T, Brochmann C, Coissac E, Taberlet P, Kauserud H. 2010. ITS as an environmental DNA barcode for fungi: an in silico approach reveals potential PCR biases. BMC Microbiology 10: 189. DOI: 10.1186/1471-2180-10-189

Bels PJ, Pataragetvit S. 1982. Edible mushrooms in Thailand cultivated by termite. In: Chang ST, Quimio TH (eds.), Tropical Mushrooms Biological Nature and Cultivation Methods. The Chinese University Press, Hong Kong.

Hajibabaei M, Singer GAC, Hebert PDN, Hickey DA. 2007. DNA barcoding: how it complements taxonomy, molecular phylogenetics and population genetics. Trends Genet 23 (4): 167-172.

Hall TA. 1999. BioEdit: A user-friendly biological sequence alignment editor and analysis program for Windows 95/98/NT. Nucleic Acids Symp Ser 41: 95-98.

Hibbett D, Abarenkov K, Kõljalg U, Öpik M, Chai B, Cole J, Wang Q, Crous P, Robert V, Helgason T, Herr JR, Kirk P, Lueschow S, O'Donnell K, Nilsson RH, Oono R, Schoch C, Smyth C, Walker DM, Porras-Alfaro A, Taylor JW, Geiser DM. 2016. Sequence-based classification and identification of Fungi. Mycologia 108 (6): 10491068 . 
Hofstetter V, Clémençon H, Vilgalys R, Moncalvo JM. 2002 Phylogenetic analyses of the Lyophylleae (Agaricales, Basidiomycota) based on nuclear and mitochondrial RDNA sequences. Mycol Res 106 (9): 1043-1059.

Hyde KD, Norphanphoun C, Chen J, Dissanayake AJ, Doilom M, Hongsanan S, Jayawardena RS, Jeewon R, Perera RH, Thongbai B, Wanasinghe DN, Wisitrassameewong K, Tibpromma S. 2018. Thailand's amazing diversity: up to $96 \%$ of fungi in northern Thailand may be novel. Fungal Divers 93: 215-239.

Karun NC, Sridhar KR. 2013. Occurrence and distribution of Termitomyces (Basidiomycota, Agaricales) in the Western Ghats and on the west coast of India. Czech Mycol 65 (2): 233-254.

Kirk PM, Cannon PF, Minter DW, Stalpers JA. 2008. Ainsworth and Bisby's Dictionary of Fungi. 10th ed. Cab International, Wallingford UK.

Kosakul T, Boasri A, Chalermpongse A, Kuhiran M. 2007. Genetic diversity of Termitomyces in central Thailand using isozyme markers. J Sci Res Chulalongkorn Univ 32: 63-72.

Kumar S., Stecher G., Tamura K.. 2016. MEGA7: Molecular evolutionary genetics analysis version 7.0 for bigger datasets. Mol Biol Evol 33 (7): 1870-1874.

Largent LD, Johnson D, Watling R. 1977. How to Identify Mushrooms to Genus III: Microscopic Features. Mad River Press Inc., Eureka. CA.

Largent LD. 1973. How to Identify Mushrooms to Genus I: Macroscopic Features. Mad River Press, Inc., California.

Marc Stadler Hussain S, Afshan NS, Ahmad H, Khalid AN. 2015. New report of edible mushroom, Termitomyces unkowaan, from Pakistan. Sylwan 159 (6): 185-197.

Mossebo DC, Njounkou AL, Piatek M, Kengni Ayissi B, Djamndo Djasbe M. 2009. Termitomyces striatus $\mathrm{f}$. pileatus $\mathrm{f}$. nov. and f. brunneus $\mathrm{f}$ nov. from Cameroon with a key to central African species. Mycotaxon 107: 315-329.

Nakamura T, Yamada KD, Tomii K, Katoh K. 2018. Parallelization of MAFFT for large-scale multiple sequence alignments. Bioinformatics 34 (14): 2490-2492.

Nobre T, Eggleton P, Aanen DK. 2010. Vertical transmission as the key to the colonization of Madagascar by fungus-growing termites? Proc Biol Sci 277 (1680): 359-365.

Olila D, Kyeyune G, Kabasa JD, Kisovi I, Munishi PKT. 2007. Assessment of potential for domestication of Termitomyces microcarpus: an indigenous edible and medicinal mushroom from Lake Victoria basin. Agric J 2: 627-631.

Otieno NC. 1964. Contributions to knowledge of termite fungi in East Africa. Proc East Afr Acad 11: 108-120.

Pegler DN, Vanhaecke M. 1994. Termitomyces of Southeast Asia. Kew Bulletin, UK.

Pitchayangkul S. 1998. Termitomyces sp. and Fusan-hybrid Mushrooms. The War Veterans Organization Printing, Bangkok. [Thai]

Raja HA, Miller AN, Pearce CJ, Oberlies NH. 2017. Fungal identification using molecular tools: a primer for the natural products research community. J Nat Prod 80 (3): 756-770.
Rouland-Lefevre C, Diouf MN, Brauman A, Neyra M. 2002. Phylogenetic relationships in Termitomyces (Family Agaricaceae) based on the nucleotide sequence of ITS: A first approach to elucidate the evolutionary history of the symbiosis between fungus-growing termites and their fungi. Mol Phylogenet Evol 22 (3): 423-429.

Sangvichien E, Taylor-Hawksworth PA. 2001. Termitomyces mushrooms: a tropical delicacy. Mycologist 15: 31-33.

Sangwanit U, Suwannarit P, Payapanon A, Luangsa-ard J, Chandrasrikul A, Sakolrak B. 2013. Checklist of mushrooms. Biodiversity-based Economy Development Office (Public Organization), Bangkok. [Thai]

Seifert KA. 2009. Progress towards DNA barcoding of fungi. Mol Ecol Resour 9: 83-89.

Siddiquee S, Yee WY, Taslima K, Fatihah NN, Kumar SV, Hasan MM. 2012. Sequence analysis of the ribosomal DNA internal transcribed spacer regions in Termitomyces heimii species. Ann Microbiol 62: 797-803.

Tanticharoen M. 2004. Introduction to Thai biodiversity. In: Jones EBG, Tantichareon M, Hyde KD (eds.). Thai Fungal Diversity. National Center for Genetic Engineering and Biotechnology (BIOTEC), Thailand.

Taprab Y, Ohkuma M, Johjima T, Maeda Y, Moriya S, Inoue T, Suwanarit P, Noparatnaraporn N, Kudo T. 2002. Molecular phylogeny of symbiotic basidiomycetes of fungus-growing termites in Thailand and their relationship with the host. Biosci Biotech Bioch 66: 1159-1163.

Taylor JW, Jacobson DJ, Kroken S, Kasuga T, Geiser DM, Hibbett DS, Fisher MC. 2000. Phylogenetic species recognition and species concepts in fungi. Fungal Genet Biol 31: 21-32.

The Royal Institute. 1996. Edible mushrooms and poisonous mushrooms in Thailand. The Royal Institute Publication, Bangkok. [Thai]

Tibuhwa DD, Kivaisi AK, Magingo FSS. 2010. Utility of the macromicromorphological Characteristics used in classifying the species of Termitomyces. Tanz J Sci 36: 31-45.

Vilgalys R, Hester M. 1990. Rapid genetic identification and mapping of enzymatically amplified ribosomal DNA from several Cryptococcus species. J Bacteriol 172 (8): 4238-4246.

Wei TZ, Tang BH, Yao YJ, Pegler DN. 2006. A revision of Sinotermitomyces, a synonym of Termitomyces (Agaricales). Fungal Divers 21: 225-237.

Wei TZ, Tang BH, Yao YJ. 2009. Revision of Termitomyces in China. Mycotaxon 108: 257-285.

Wei TZ, YAO YJ, Wang B, Pegler DN. 2004. Termitomyces bulborhizus sp. nov. from China, with a key to allied species. Mycol Res 108 (12): 1458-1462.

White TJ, Bruns T, Taylor J. 1990. Amplification and direct sequencing of fungal ribosomal RNA genes for phylogenetics. In: Innis MA, Gelfand DH, Sninsky JJ, White JW (eds.). A Guide to Molecular Methods and Applications. Academic Press, New York.

Xu J, Adamowicz S. 2016. Fungal DNA barcoding. Genome 59: 913-932. 